\title{
Cathodoluminescence of Single Disk-Like ZnO Prepared by Low Temperature Solution-Based Method*
}

\author{
Gui Han,$^{\dagger}$ Morihiro Okada, Zhiyan Xiao, Yoichiro Neo, Toru Aoki, and Hidenori Mimura ${ }^{\ddagger}$ \\ Research Institute of Electronics, Shizuoka University, \\ 3-5-1 Johoku, Naka-ku, Hamamatsu 432-8011, Japan \\ Hideyuki Watanabe and Kazunori Hikosaka \\ Advanced Industrial Science and Technology, Central 2, Umezono, Tsukuba 305-8568, Japan
}

(Received 2 June 2008; Accepted 8 December 2008; Published 4 April 2009)

\begin{abstract}
Single crystalline $\mathrm{ZnO}$ disk-like structure with good luminescent properties was obtained by a low temperature solution based method. Micro-emulsion was employed to assist the shape control. The product has a perfect hexagonal shape with flat bottom and up surfaces, and has a wide size distribution, the size ranges from nano to micro-scale. Electron diffraction (ED) pattern coupled with transmission electron microscopy (TEM) indicate that the single disk was single-crystalline. In cathodoluminescence (CL) studies at room temperature for wavelengths between 350 and $675 \mathrm{~nm}$, we have observed a single exciton peak around $390 \mathrm{~nm}$ without any deep-level emission. The monochromatic CL mapping in the ultra-violet (UV) region has such properties as: the intensity is concentrated along the boundary of the cavity; the emission is predominantly at the corner.
\end{abstract}

[DOI: $10.1380 /$ ejssnt.2009.354]

Keywords: $\mathrm{ZnO}$; Cathodoluminescence; Microdisk; Whispering-gallery-mode

\section{INTRODUCTION}

$\mathrm{ZnO}$ has a wide and direct band gap of $3.37 \mathrm{eV}$ with the large exciton binding energy of $60 \mathrm{meV}$ at room temperature. In recent years, great interests are focused on nanostructural zinc oxide because can be widely used in UV-laser with low threshold, field emission array, surface acoustic device, transparent thin film transistors [1].

Many researchers have focused on producing onedimensional (1D) nano-materials, and various types of morphologies in the nano- and micro- scale have already been synthesized, including rods, wires, needles, belts, flower-like, and tube-like $\mathrm{ZnO}$ structures [2]. Onedimensional $\mathrm{ZnO}$ nanostuctures are easily synthesized owing to a facile growth along the $c$ axis of the wurtzite crystal, which has a hexagonal unit cell with six nonpolar $\{10 \overline{1} 0\}$ prismatic faces capped by polar oxygen $(000 \overline{1})$ and zinc (0001) basal planes. The polar faces are electro statically unstable Tasker type III surfaces, making the $\{0001\}$ planes have the highest energy of the low-index surfaces [3]. In contrast to one-dimensional nanostructures, disk-shaped morphology is less known, few studies on hexagonal $\mathrm{ZnO}$ disk like structures have been reported [4-7].

For the fabrication of hexagonal disk-like $\mathrm{ZnO}$ materials, to our knowledge, two methods have been developed: vapor phase transport (VPT) $[4,7]$ and solution based methods $[5,6]$. The solution method has several advantages over VPT, such as large scale production, low temperature, easy to control and good reproducibility. In the case of solution-phase syntheses, the growth rates of various surfaces can also be kinetically controlled, at moderate temperatures. For example, the growth of certain

\footnotetext{
*This paper was presented at the 14th International Conference on Solid Films and Surfaces (ICSFS-14), Trinity College Dublin, Ireland, 29 June - 4 July, 2008.

$\dagger$ Corresponding author: hanguihq@hotmail.com

$\ddagger$ Corresponding author: mimura@rie.shizuoka.ac.jp
}

surfaces can be impeded by using additives that preferentially adsorb to specific crystal faces $[5,6]$.

In the present study, we are focusing on the preparation of high crystalline hexagonal $\mathrm{ZnO}$ micro-disk and the main optical properties of a single disk were characterized with high resolution SEM coupled cathodoluminescence. With the change of starting material from ammonia to HMT (hexamethylenetetramine), the quality of the asprepared sample was improved, the crystal has fewer defects like low-angle grain boundaries and the thickness of the sample was uniform towards the center.

\section{EXPERIMENTAL}

The fabrication of the $\mathrm{ZnO}$ microdisks was performed by the low temperature solution-based method similar to ref. [5] with change of the starting material from ammonia to HMT (hexamethylenetetramine, $\mathrm{C}_{6} \mathrm{H}_{12} \mathrm{~N}_{4}$ ). In a typical process: first, three kind of solution was prepared, namely $0.10 \mathrm{M}$ of $\mathrm{Zn}\left(\mathrm{NO}_{3}\right)_{2}, 0.10 \mathrm{M}$ of HMT in water solution, and $0.10 \mathrm{M}$ sodium bis (2-ethylhexyl) sulfosuccinate (AOT) in 1-butanol solution. Next, AOT microemulsion was prepared by adding mixed $50 \mathrm{ml} \mathrm{Zn}\left(\mathrm{NO}_{3}\right)_{2}$ and $50 \mathrm{ml}$ HMT solution to $10 \mathrm{ml}$ AOT solution and vigorously stirring for 3 hours. Then, the mixture was transferred to a Teflon lined autoclave and kept at $90^{\circ} \mathrm{C}$ for 12 hours. A white suspension was obtained and centrifuged to separate the precipitate, which was washed several times with distilled water and absolute ethanol. Finally, white powder was obtained by drying at $60^{\circ} \mathrm{C}$ in atmosphere.

The crystal structure and morphology of the asprepared samples were characterized by x-ray diffraction (XRD, Rigaku D/max 2200V, Cu-K $\alpha$ ), transmission electron microscopy (TEM) and field emission scanning electron microscopy (FE-SEM, LEO SUPER 35) studies. The SEM system also has cathodoluminescence (CL) and energy dispersive x-ray analysis (EDX) attachments. 


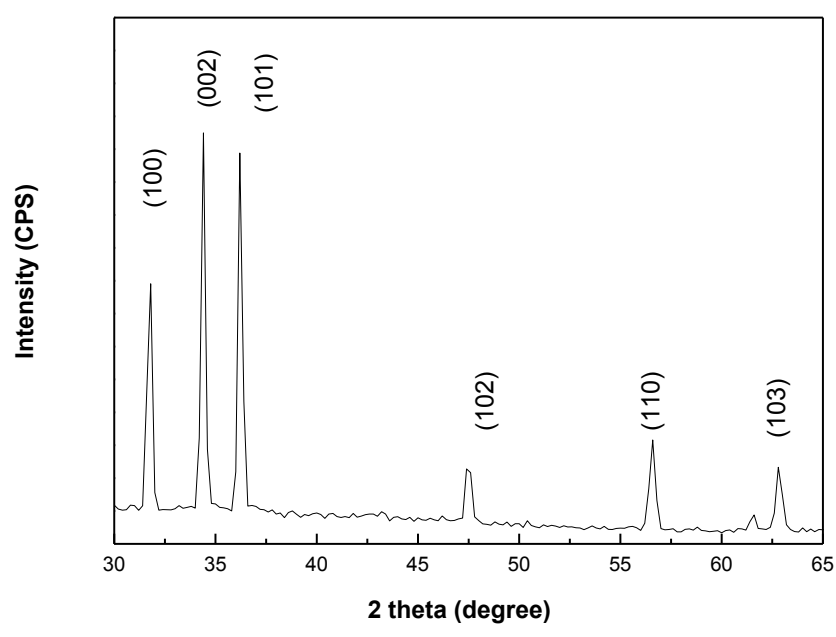

FIG. 1: XRD pattern of the as-prepared sample. All the crystal surfaces are indexed (PDF \# 36-1451).

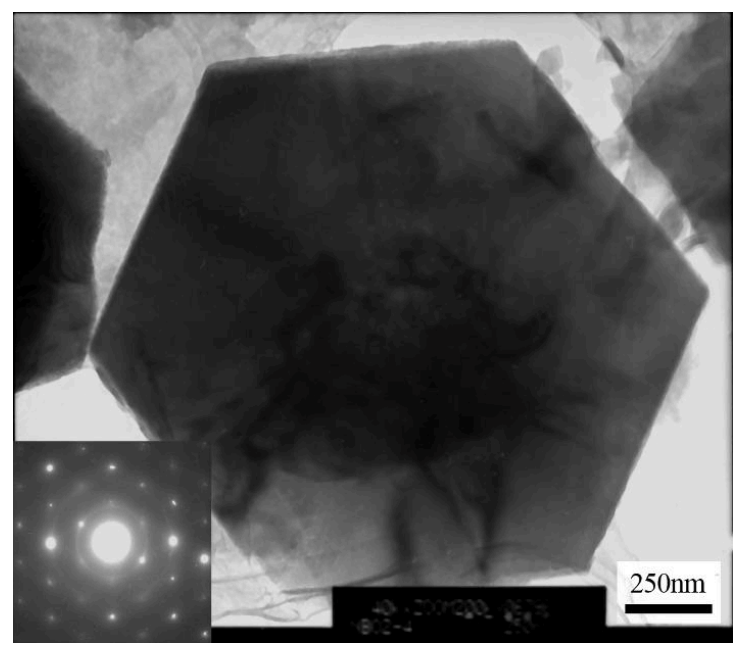

FIG. 2: TEM image of the single $\mathrm{ZnO}$ disk. The insertion shows the corresponding ED pattern.

\section{RESULTS AND DISCUSSIONS}

A XRD pattern of the sample is illustrated in Fig. 1. All the diffraction peaks can be readily indexed as typical wurtzite hexagonal phase of $\mathrm{ZnO}$ with lattice constants of $a=3.250 \AA$ and $c=5.206 \AA$ in PDF No. 36-1451. No peaks of other impurities were detected in the spectrum, indicating that the as-prepared samples are pure $\mathrm{ZnO}$.

TEM and a corresponding electron diffraction (ED) pattern of the $\mathrm{ZnO}$ disk are shown in Fig. 2. The single disk is about $1.6 \mu \mathrm{m}$ in diameter and has no cracks such as low-angle grain boundaries as shown in ref. [5], and the thickness toward the center are uniform. The wurtzite crystal structure can also be confirmed by the ED pattern in the insertion of Fig. 2, which has six-fold symmetry.

Morphologies of the as-synthesized disk-like samples observed by SEM are depicted in Fig. 3(a) and 3(c). The hexagonal zinc oxide crystal has wurtzite (hcp) structure, and has both polar and non-polar faces. The bottom and up surface are either Zn-terminated (the (0001) face) or O-terminated (the $(000 \overline{1})$ face). The six side surfaces include $(11 \overline{2} 0)$ and $(10 \overline{1} 0)$ faces.

The surface of the single disk shown in the SEM images was also tested by the energy dispersive x-ray (EDX); and the spectra (not shown here) confirmed that the composition of the microdisk was $\mathrm{ZnO}$, and the atomic ratio of $\mathrm{Zn}$ and $\mathrm{O}$ was 1.12: 1 . The samples have a large size distribution, and the size of the particulate varies from $500 \mathrm{~nm}$ to several micrometers. The samples have both flat bottom and up surfaces, while the sample in ref. [5] only has one flat surface and rounded on the other surface. This may be due to different crystal growth mechanism. The morphology of a particular crystal is determined by the slowest growing faces. It is well known that the $\mathrm{c}$ axis is the fastest growing direction for $\mathrm{ZnO}$, but the growth rates of various surfaces can be kinetically controlled, especially in the case of solution-phase syntheses at moderate temperatures $[8,9]$. We have done these experiments for reference, for example, without any additives, wireand rod- like structures could be obtained by zinc nitrate and HMT; when adding sodium citrate, platelet- and rodlike structures with decreased length could be prepared. Although sodium citrate can be used to control the disk shape growth, it is not so easily controlled. In the case of AOT micro-emulsion, which has been used as a soft colloidal template for the controlling the size and shape of inorganic nanocrystals [10]. At certain concentrations, the surfactant (AOT) can self-assembly into planar lamellar phase. It was thought that the planar lamellar phase acted as a template directing the $\mathrm{ZnO}$ crystal growth and changed the growth rate of different crystal surfaces, thus the disk-like micro crystal can be formed.

HMT provides a slow controlled supply of $\mathrm{OH}-$ and is a poor ligand for zinc [8]. The following reactions, which have taken place in the solution, are pivotal in the formation of $\mathrm{ZnO}$ hexagonal disk under the AOT formed template:

$$
\begin{aligned}
\mathrm{C}_{6} \mathrm{H}_{12} \mathrm{~N}_{4} & +6 \mathrm{H}_{2} \mathrm{O} \rightarrow 6 \mathrm{HCHO}+4 \mathrm{NH}_{3} \\
\mathrm{NH}_{3} & +\mathrm{H}_{2} \mathrm{O} \leftrightarrow \mathrm{NH}_{4}^{+}+\mathrm{OH}^{-} \\
\mathrm{Zn}^{2+} & +2 \mathrm{OH}^{-} \leftrightarrow \mathrm{Zn}(\mathrm{OH})_{2} \leftrightarrow \mathrm{ZnO}(\mathrm{s})+\mathrm{H}_{2} \mathrm{O}
\end{aligned}
$$

With the slowly release of $\mathrm{OH}-$ and with the AOT formed lamellar template, the crystal with disk like shape was successfully obtained.

The hexagonal $\mathrm{ZnO}$ has potential applications in the field of optical cavities, so we performed CL measurement on two samples with diameter of $2 \mu \mathrm{m}$ and $5 \mu \mathrm{m}$. The $2 \mu \mathrm{m}$ and $5 \mu \mathrm{m}$ samples were excited by the acceleration voltage of $10 \mathrm{kV}$ and $20 \mathrm{kV}$ at room temperature, respectively. The monochromic CL mapping at a wavelength of 390nm was shown in Fig. 3(b) and 3(d), respectively. The theoretical simulation done by Wiersig [11] shows the near-field intensity pattern of the hexagonal resonant cavity, which has the following properties: the light is concentrated along the boundary of the disk; and the emission is predominantly at the corners. The images (Figs. 3(c) and $3(\mathrm{~d})$ ) taken by the monochromic mapping of our sample show almost the same properties, which was in accordance with the Fig. 3 of Kim et al. [6]. Unfortunately, we could not observe the CL intensity modulated by the resonance modes as a clear evidence for Whispering-Gallery-Mode 

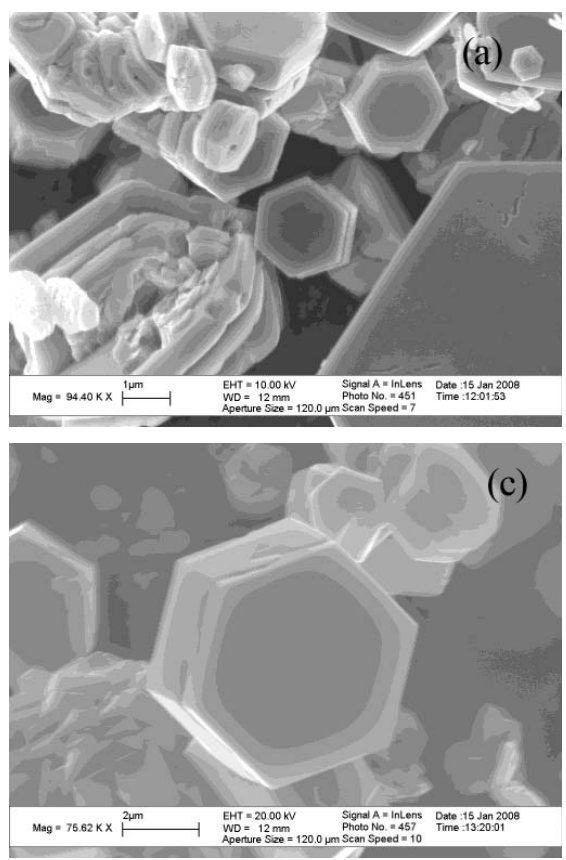

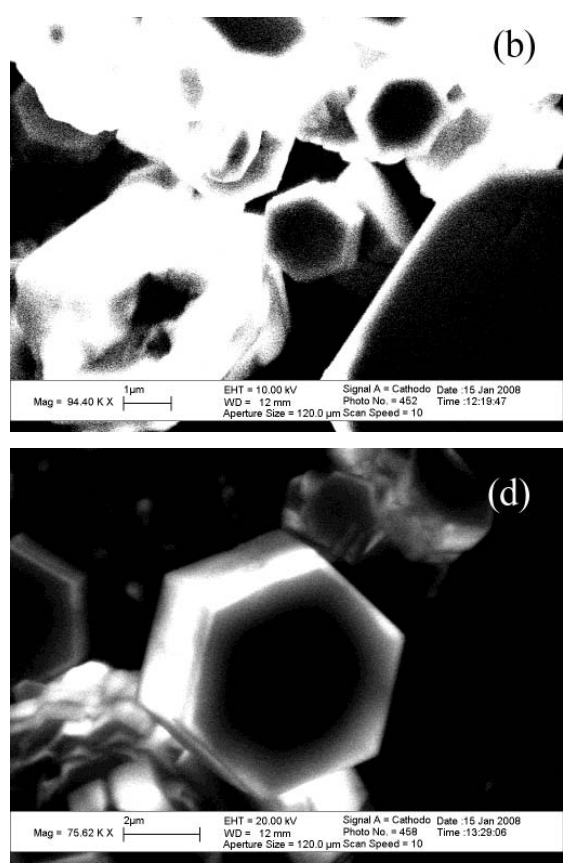

FIG. 3: (a) and (c) refer to SEM images of the $2 \mu \mathrm{m}$ and $5 \mu \mathrm{m}$ samples, respectvely. (b) and (d) refer to corresponding monochromic CL mappings. The acceleration voltages were $10 \mathrm{kV}$ and $20 \mathrm{kV}$ for the $2 \mu \mathrm{m}$ and $5 \mu \mathrm{m}$ samples, respectively.

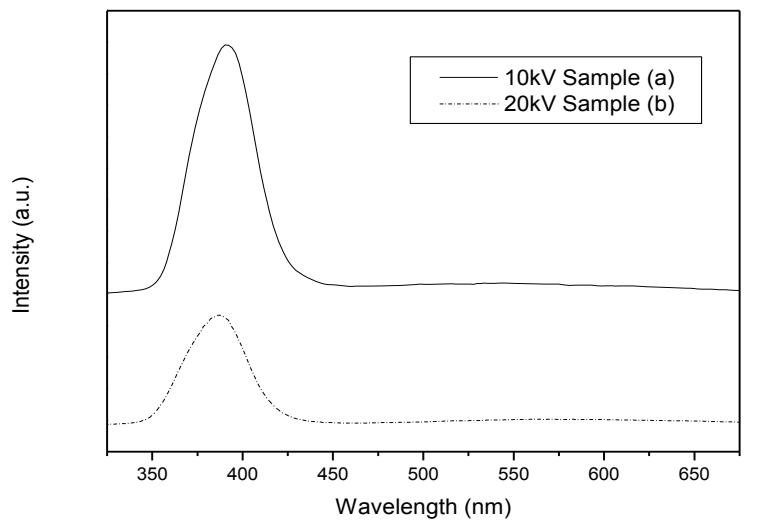

FIG. 4: Single disk CL spectra of the $2 \mu \mathrm{m}$ (a) and $5 \mu \mathrm{m}$ (b) samples under the acceleration voltages of $10 \mathrm{kV}$ and $20 \mathrm{kV}$, respectively.

(WGM). One of main reasons is due to the line broadening of WGM. The theory predicts line broadening by loss processes due to boundary wave that are scattered out the resonator and due to light paths whose angle of incidence is slightly deviating from $60^{\circ}[12]$. Additionally, the loss due to surface roughness of the cavity faces could also lead to line broadening [6].

It is known that $\mathrm{ZnO}$ displays three major peaks: an UV near-band-edge emission peak around $380 \mathrm{~nm}$, a green emission around $510 \mathrm{~nm}$, and a red emission around 650 $\mathrm{nm}$. It is generally accepted that the green and red emissions are associated with oxygen vacancies and interstitial $\mathrm{Zn}$ ions in the $\mathrm{ZnO}$ lattice. Figure 4 shows the CL spectra from a single disk, which has only one peak referred to the band gap emission at about $390 \mathrm{~nm}$, and there is no yellow luminescence related to deep levels or other visible emissions. One common view of evaluating the concentration of structural defects in $\mathrm{ZnO}$ is to compare the relative photoluminescence (PL) intensity ratio of the UV nearband-edge emission to the deep-level emissions [13]. So it can also be seen that the crystal has been well crystallized as indicated by the TEM and SEM images.

\section{CONCLUSIONS}

Undoped single crystalline hexagonal disk-like wurzite structures have been prepared by microemulsion assisted solution based method at the temperature as low as $90^{\circ} \mathrm{C}$. With HMT as reactant, the crystal quality has been improved. The crystals have fewer defects as indicated by the high ratio of the UV to visible emissions. The CL mapping in the UV region showed that luminescence was spatially localized near the boundary of the microdisk. Future work need to be done to control the scale and thickness of the disk, to get narrow size distribution, and to get clear evidence of the WGM emission.

\section{Acknowledgments}

This work is supported, in part, by Shizuoka University 21st Center of Excellence (COE) Program and a GrantAid for Scientific research from the Ministry of Education, Culture, Sports, Science and technology, Japan, and the Futaba Electronics Memorial Foundation. 
[1] Ü. Özgür, Ya. I. Alivov, C. Liu, A. Teke, M. A. Reshchikov, S. Doan, V. Avrutin, S.-J. Cho, and H. Morkoc, J. Appl. Phys. 98, 041301 (2005).

[2] M. Law, J. Goldberger, and P. D. Yang, Annu. Rev. Mater. Res. 34, 83 (2004).

[3] L. E. Greene, B. D. Yuhas, M. Law, D. Zitoun, and P. D. Yang, Inorg. Chem. 45, 7535 (2006).

[4] C. X. Xu, X. W. Sun, Z. L. Dong, and M. B. Yu, Appl. Phys. Lett. 85, 3878 (2004).

[5] F. Li, Y. Ding, P. Gao, X. Xin, and Z. L. Wang, Angew. Chem. 116, 5350 (2004).

[6] C. Kim, Y. J. Kim, E. S. Jang, G. C. Yi, and H. H. Kim, Appl. Phys. Lett. 88, 93104 (2006).

[7] D. S. Yu, Y. Chen, B. Li, X. Chen, M. Zhang, F. Zhao, and S. Ren, Appl. Phys. Lett. 91, 91116 (2007).

[8] K. Govender, D. S. Boyle, P. B. Kenway, and P. O'Brien, J. Mater. Chem. 14, 2575 (2004).

[9] Z. R. Tian, J. A. Voigt, J. Liu, B. Mckenzie, M. J. Mcdermott, M. A. Rodriguez, H. Konishi, and H. Xu, Nat. Mater. 2, 821 (2003).

[10] M. P. Pileni, Nat. Mater. 2, 145 (2003).

[11] J. Wiersig, Phys. Rev. A 67, 23807 (2003).

[12] X. Liu, W. Fang, Y. Huang, X. H. Wu, S. T. Ho, H. Cao, and R. P. H. Chang, Appl. Phys. Lett. 84, 2488 (2004).

[13] Y. Chen, D. M. Bagnall, H. Koh, K. Park, K. Hiraga, Z. Zhu, and T. Yao, J. Appl. Phys. 84, 3912 (1998). 